\title{
Spin dynamics in gravitational fields of rotating bodies and the equivalence principle
}

\author{
Yuri N. Obukhov* \\ Department of Mathematics, University College London, \\ Gower Street, London, WC1E 6BT, UK \\ Alexander J. Silenkot \\ Research Institute of Nuclear Problems, \\ Belarusian State University, Minsk 220080, Belarus \\ Oleg V. Teryaevt \\ Bogoliubov Laboratory of Theoretical Physics, \\ Joint Institute for Nuclear Research, Dubna 141980, Russia
}

\begin{abstract}
We discuss the quantum and classical dynamics of a particle with spin in the gravitational field of a rotating source. A relativistic equation describing the motion of classical spin in curved spacetimes is obtained. We demonstrate that the precession of the classical spin is in a perfect agreement with the motion of the quantum spin derived from the Foldy-Wouthuysen approach for the Dirac particle in a curved spacetime. We show that the precession effect depends crucially on the choice of a tetrad. The results obtained are compared to the earlier computations for different tetrad gauges.

PACS numbers: 04.20.Cv; 04.80.Cc; 03.65.Sq
\end{abstract}

${ }^{*}$ Electronic address: yo@thp.Uni-Koeln.DE

${ }^{\dagger}$ Electronic address: silenko@inp.minsk.by

${ }^{\ddagger}$ Electronic address: teryaev@thsun1.jinr.ru 


\section{INTRODUCTION}

A rotation of a central body, defining a difference between stationary and static spacetimes, leads to an appearance of specific gravitational effects. The most important effect has been predicted by Lense and Thirring [1]. It consists in frame dragging around rotating bodies and is manifested in a precession of satellite orbits and gyroscopes (i.e. classical spins). The nonrelativistic formula for the latter effect has been derived by L. Schiff [2] (and further refined and generalized in [3] ).

In the present work, we analyze quantum and classical spins in stationary spacetimes. We use the weak-field approximation when all components of the metric tensor $g_{i j}$ are close to the corresponding components of the Minkowski tensor $\eta_{i j}\left(\left|h_{i j}\right| \equiv\left|g_{i j}-\eta_{i j}\right| \ll 1\right)$. The formulas calculated for classical spins extend the previously obtained results to the relativistic case. The investigation of the quantum dynamics of spins is carried out for the first time.

The theory of classical spin in the first-order (linear) approximation can be formulated as follows. A particle is characterized by its position in spacetime, $x^{i}(\tau)$ which is a function of the proper time $\tau$, and by the 4 -vector of spin $S^{\alpha}$. The 4 -velocity of a particle $U^{\alpha}=e_{i}^{\alpha} d x^{i} / d \tau$ is normalized by the condition $g_{\alpha \beta} U^{\alpha} U^{\beta}=c^{2}$ where $g_{\alpha \beta}=\operatorname{diag}\left(c^{2},-1,-1,-1\right)$ is the flat Minkowski metric. In order to be able to describe spinning particles both in flat and curved spacetime (as well as in arbitrary curvilinear coordinates), we use the tetrad $e_{i}^{\alpha}$ to transform the components of different objects from the coordinate basis (associated with the local coordinates $x^{i}$ ) to a local orthonormal frame. When the gravitational field is absent, it is possible to choose the Cartesian coordinates everywhere and use the holonomic orthonormal frame which coincides with the natural frame, so that $e_{i}^{\alpha}=\delta_{i}^{\alpha}$ then. In general, the tetrad coefficients satisfy $g_{\alpha \beta} e_{i}^{\alpha} e_{i}^{\beta}=g_{i j}$ for an arbitrary spacetime metric $g_{i j}$.

The foundations of the classical theory of particles with spin were laid down by Mathisson and Papapetrou [4, 5] (for a review see [6], e.g.). Pomeransky, Khriplovich [7] and Dvornikov [8] developed the relativistic approach for the equation of motion defining the dynamics of three-component physical spin in curved spacetimes. This equation perfectly describes the dynamics of the spin in static spacetimes. Here we present a rigorous deduction of the equation of motion of the three-component spin, confirming the heuristic arguments of [7]. At the same time, we show here that this equation can be used for nonstatic spacetimes 
only with the special choice of tetrads satisfying the condition $e_{\widehat{a}}^{0}=0$.

In this paper, we consider the two important problems. One aim is to generalize the methods of the Foldy-Wouthuysen transformations, that we previously used for the analysis of the spin in static gravitational fields, to the case of the stationary gravitational configurations. Another aim is to systematically investigate the dependence of the spin dynamics on the choice of a tetrad. In particular, we derive the general result for the angular velocity of spin precession that is valid for an arbitrary tetrad gauge.

The paper is organized as follows. In Sec. II we consider the Dirac equation in a weak gravitational field of a rotating source. The Hermitian Hamiltonian is derived. Sec. III presents the derivation of the precession angular velocity of spin in a stationary gravitational field. The dynamics of a classical spin is analyzed in Sec. IV] where we find a general expression for the precession velocity of the physical spin in arbitrary external classical fields. These results are then applied in Sec. $\nabla$ to the derivation of the classical spin dynamics in arbitrary gravitational field configurations. Sec. VI is devoted to the analysis of the dependence of the spin precession effect on the choice of a tetrad. We find a general equation that makes it possible to directly compare results obtained in the literature in different tetrad gauges. Specifically, we demonstrate the complete agreement of the classical and quantum spin dynamics in the Schwinger gauge. In Sec. VII we show that our general results can be also used for the study of the motion of spin in the flat spacetime for the rotating reference frame. Our derivations confirm the earlier observations obtained on the basis of the Thomas precession arguments. Finally, in Sec. VIII we draw the conclusions.

We denote world indices by Latin letters $i, j, k, \cdots=0,1,2,3$ and reserve first Greek letters for tetrad indices, $\alpha, \beta, \cdots=0,1,2,3$. Spatial indices are denoted by Latin letters from the beginning of the alphabet, $a, b, c, \cdots=1,2,3$. The separate tetrad indices are distinguished by hats. 


\section{DIRAC HAMILTONIAN FOR A STATIONARY METRIC}

The approximate gravitational field of a rotating body at a large distance is described by the Lense-Thirring (LT) metric [1]:

$$
\begin{aligned}
d s^{2}= & {\left[1-\frac{2 G M}{c^{2} \rho}\right] c^{2} d t^{2}-a \sin ^{2} \theta \frac{4 G M}{c^{2} \rho} c d t d \phi } \\
& -\left[1+\frac{2 G M}{c^{2} \rho}\right] d \rho^{2}-\rho^{2}\left[d \theta^{2}+\sin ^{2} \theta d \phi^{2}\right] .
\end{aligned}
$$

With the help of the coordinate transformation

$$
\rho=r\left(1+\frac{G M}{2 c^{2} r}\right)^{2},
$$

one can bring the line element to the isotropic form and subsequently use the Cartesian coordinate system. The final form of the line element is given by

$$
d s^{2}=V^{2} c^{2} d t^{2}-W^{2} \delta_{a b}\left(d x^{a}-K^{a} c d t\right)\left(d x^{b}-K^{b} c d t\right)
$$

with

$$
\begin{aligned}
V & =\left(1-\frac{G M}{2 c^{2} r}\right)\left(1+\frac{G M}{2 c^{2} r}\right)^{-1}, \\
W & =\left(1+\frac{G M}{2 c^{2} r}\right)^{2}, \\
K^{a} & =\frac{1}{c} \epsilon^{a b c} \omega_{b} x_{c} .
\end{aligned}
$$

The non-diagonal components of the metric (that reflect the rotation of the source) are described by the so-called Kerr field $\boldsymbol{K}$ that is given by Eq. (6) with

$$
\boldsymbol{\omega}=\frac{2 G}{c^{2} r^{3}} \boldsymbol{J}=\left(\begin{array}{ll}
0, & 0, \quad \frac{2 G M a}{c r^{3}}
\end{array}\right),
$$

where $\boldsymbol{J}=M c a \boldsymbol{e}_{z}$ is the total angular momentum of the source.

The exact metric of the flat spacetime seen by an accelerating and rotating observer also has form (3). In the latter case [9], however,

$$
V=1+\frac{\boldsymbol{a} \cdot \boldsymbol{r}}{c^{2}}, \quad W=1, \quad K^{a}=-\frac{1}{c}(\boldsymbol{\omega} \times \boldsymbol{r})^{a},
$$

where $\boldsymbol{a}$ describes acceleration of the observer and $\boldsymbol{\omega}$ is an angular velocity of a noninertial reference system. Both are independent of the spatial coordinates, but may depend arbitrarily on time $t$. 
The similarity between the two cases is not occasional. Lense and Thirring have discovered in 1918 that rotating bodies "drag" the spacetime around themselves (frame dragging [1]). In other words, they have demonstrated the equivalence between rotating frames and spacetimes created by rotating bodies. In the weak-field approximation, the motion of particles in a gravitational field of a rotating source is identical to their motion in a noninertial frame rotating with the angular velocity (see, e.g., Ref. [10])

$$
\boldsymbol{\omega}=\frac{c}{2} \operatorname{curl} \boldsymbol{g}, \quad g_{a}=-g_{0 a} .
$$

Let us choose the orthonormal tetrad

$$
e_{i}^{\widehat{0}}=V \delta_{i}^{0}, \quad e_{i}^{\widehat{a}}=W\left(\delta_{i}^{a}-K^{a} \delta_{i}^{0}\right), \quad a, b=1,2,3 .
$$

The covariant Dirac equation for spin-1/2 particles in curved spacetimes has the form

$$
\left(i \hbar \gamma^{\alpha} D_{\alpha}-m c\right) \psi=0, \quad \alpha=0,1,2,3
$$

The Dirac matrices $\gamma^{\alpha}$ are defined in local Lorentz (tetrad) frames. The spinor covariant derivatives are given by

$$
D_{\alpha}=e_{\alpha}^{i} D_{i}, \quad D_{i}=\partial_{i}+\frac{i}{4} \sigma_{\alpha \beta} \Gamma_{i}^{\alpha \beta},
$$

where $\Gamma_{i}^{\alpha \beta}=-\Gamma_{i}^{\beta \alpha}$ are the Lorentz connection coefficients, $\sigma^{\alpha \beta}=i\left(\gamma^{\alpha} \gamma^{\beta}-\gamma^{\beta} \gamma^{\alpha}\right) / 2$. Eqs. (11),(12) show that the gravitational and inertial effects are encoded in coframes (see Refs. [11, 12] and references therein).

Eq. (11) is recast into the familiar Schrödinger form

$$
i \hbar \frac{\partial \psi}{\partial t}=\mathcal{H} \psi
$$

with the Hamilton operator

$$
\begin{aligned}
\mathcal{H}= & \beta m c^{2} V+\frac{V}{W} c(\boldsymbol{\alpha} \cdot \boldsymbol{p})-\frac{i \hbar c}{2 W}(\boldsymbol{\alpha} \cdot \boldsymbol{\nabla} V)-\frac{i \hbar c V}{W^{2}}(\boldsymbol{\alpha} \cdot \boldsymbol{\nabla} W) \\
& -i \hbar c \boldsymbol{K} \cdot \boldsymbol{\nabla}-\frac{i \hbar c}{2}(\boldsymbol{\nabla} \cdot \boldsymbol{K})-\frac{3 i \hbar c}{2 W}(\boldsymbol{K} \cdot \boldsymbol{\nabla} W)+\frac{\hbar c}{4}(\boldsymbol{\nabla} \times \boldsymbol{K}) \cdot \boldsymbol{\Sigma} .
\end{aligned}
$$

Here $\boldsymbol{p}=-i \hbar \nabla$, and we remind that $\beta=\gamma^{\hat{0}}, \boldsymbol{\alpha}=\left\{\alpha^{a}\right\}, \boldsymbol{\Sigma}=\left\{\Sigma^{a}\right\}$, where the 3-vector-valued Dirac matrices have their usual form: $\alpha^{a}=\gamma^{\hat{0}} \gamma^{a}$ and $\Sigma_{a}=i \epsilon_{a b c} \gamma^{b} \gamma^{c} / 2(a, b, c, \cdots=1,2,3)$. Redefining the spinor field and the Hamiltonian,

$$
\psi^{\prime}=W^{3 / 2} \psi
$$


we obtain the new Hamiltonian (which is explicitly Hermitian with respect to the usual flat space scalar product):

$$
\begin{aligned}
\mathcal{H}^{\prime}= & \beta m c^{2} V+\frac{c}{2}[(\boldsymbol{\alpha} \cdot \boldsymbol{p}) \mathcal{F}+\mathcal{F}(\boldsymbol{\alpha} \cdot \boldsymbol{p})] \\
& +\frac{c}{2}(\boldsymbol{K} \cdot \boldsymbol{p}+\boldsymbol{p} \cdot \boldsymbol{K})+\frac{\hbar c}{4}(\boldsymbol{\nabla} \times \boldsymbol{K}) \cdot \boldsymbol{\Sigma} .
\end{aligned}
$$

Here $\mathcal{F}=V / W$.

Substituting (4)-(7) into (16), we find:

$$
\begin{aligned}
\mathcal{H}^{\prime}= & \beta m c^{2} V+\frac{c}{2}[(\boldsymbol{\alpha} \cdot \boldsymbol{p}) \mathcal{F}+\mathcal{F}(\boldsymbol{\alpha} \cdot \boldsymbol{p})] \\
& +\frac{2 G}{c^{2} r^{3}} \boldsymbol{J} \cdot(\boldsymbol{r} \times \boldsymbol{p})+\frac{\hbar G}{2 c^{2} r^{3}}\left[\frac{3(\boldsymbol{r} \cdot \boldsymbol{J})(\boldsymbol{r} \cdot \boldsymbol{\Sigma})}{r^{2}}-\boldsymbol{J} \cdot \boldsymbol{\Sigma}\right] .
\end{aligned}
$$

Note that the angular momentum operator $\boldsymbol{l}=\boldsymbol{r} \times \boldsymbol{p}$ commutes with $\boldsymbol{\omega}$ which depends on the radius. Dirac Hamiltonian (17) contains the first part describing the static gravitational field and the second one characterizing the contribution of rotation of the central body.

\section{FOLDY-WOUTHUYSEN HAMILTONIAN AND OPERATOR EQUATIONS OF MOTION}

To obtain the FW Hamiltonian, we perform the FW transformation by the method developed in Refs. [13, 14]. In the weak field approximation, there are three small parameters:

$$
|V-1| \ll 1, \quad|\mathcal{F}-1| \ll 1, \quad|\boldsymbol{K}| \ll 1 .
$$

Evidently, any bilinear combinations of these parameters can be neglected. The FW Hamiltonian can be presented as a sum of a free particle Hamiltonian and terms proportional to $|V-1|,|\mathcal{F}-1|$, and $\boldsymbol{K}$. Only the last term, $\mathcal{H}_{F W}^{(2)}$, defines the contribution of rotation of the central body, while the other terms characterize the FW Hamiltonian of the particle in a static gravitational field. The rotation-independent contribution $\mathcal{H}_{F W}^{(1)}$ was calculated earlier [15]:

$$
\begin{gathered}
\mathcal{H}_{F W}^{(1)}=\beta \epsilon+\frac{\beta}{2}\left\{\frac{m^{2} c^{4}}{\epsilon}, V-1\right\}+\frac{\beta}{2}\left\{\frac{c^{2} \boldsymbol{p}^{2}}{\epsilon}, \mathcal{F}-1\right\}-\frac{\beta \hbar m c^{4}}{4 \epsilon\left(\epsilon+m c^{2}\right)}[\boldsymbol{\Sigma} \cdot(\boldsymbol{\phi} \times \boldsymbol{p}) \\
-\boldsymbol{\Sigma} \cdot(\boldsymbol{p} \times \boldsymbol{\phi})+\hbar \nabla \cdot \boldsymbol{\phi}]+\frac{\beta \hbar^{2} m c^{6}\left(2 \epsilon^{3}+2 \epsilon^{2} m c^{2}+2 \epsilon m^{2} c^{4}+m^{3} c^{6}\right)}{8 \epsilon^{5}\left(\epsilon+m c^{2}\right)^{2}}(\boldsymbol{p} \cdot \nabla)(\boldsymbol{p} \cdot \boldsymbol{\phi}) \\
+\frac{\beta \hbar c^{2}}{4 \epsilon}[\boldsymbol{\Sigma} \cdot(\boldsymbol{f} \times \boldsymbol{p})-\boldsymbol{\Sigma} \cdot(\boldsymbol{p} \times \boldsymbol{f})+\hbar \nabla \cdot \boldsymbol{f}]-\frac{\beta \hbar^{2} c^{4}\left(\epsilon^{2}+m^{2} c^{4}\right)}{4 \epsilon^{5}}(\boldsymbol{p} \cdot \nabla)(\boldsymbol{p} \cdot \boldsymbol{f}) .
\end{gathered}
$$


Here $\epsilon=\sqrt{m^{2} c^{4}+c^{2} \boldsymbol{p}^{2}}$ and the curly bracket $\{\cdots, \cdots\}$ denotes the anticommutator. We also use the notation of [11, 15] for the gradients: $\boldsymbol{\phi}=\left\{\partial_{a} V\right\}, \boldsymbol{f}=\left\{\partial_{a} \mathcal{F}\right\}, a=1,2,3$.

To find the rotation-dependent term $\mathcal{H}_{F W}^{(2)}$, it is sufficient to keep the leading term in the FW transformation operator [15] corresponding to the free particle transformation:

$$
U=\frac{\epsilon+m c^{2}+\beta c \boldsymbol{\alpha} \cdot \boldsymbol{p}}{\sqrt{2 \epsilon\left(\epsilon+m c^{2}\right)}} .
$$

Corrections to this approximation can be neglected because they only affect terms in the FW Hamiltonian which are bilinear in small parameters $|V-1|,|\mathcal{F}-1|$, and $\boldsymbol{K}$.

This FW transformation leads after straightforward but tedious calculations to the final FW Hamiltonian which is given by

$$
\begin{gathered}
\mathcal{H}_{F W}=\mathcal{H}_{F W}^{(1)}+\mathcal{H}_{F W}^{(2)}, \quad \mathcal{H}_{F W}^{(2)}=\frac{2 G}{c^{2} r^{3}} \boldsymbol{J} \cdot \boldsymbol{l}+\frac{\hbar G}{2 c^{2} r^{3}}\left[\frac{3(\boldsymbol{r} \cdot \boldsymbol{J})(\boldsymbol{r} \cdot \boldsymbol{\Sigma})}{r^{2}}-\boldsymbol{J} \cdot \boldsymbol{\Sigma}\right] \\
-\frac{3 \hbar G}{8}\left\{\frac{1}{\epsilon\left(\epsilon+m c^{2}\right)},\left[\frac{2\{(\boldsymbol{J} \cdot \boldsymbol{l}),(\boldsymbol{\Sigma} \cdot \boldsymbol{l})\}}{r^{5}}+\frac{1}{2}\left\{(\boldsymbol{\Sigma} \cdot(\boldsymbol{p} \times \boldsymbol{l})-\boldsymbol{\Sigma} \cdot(\boldsymbol{l} \times \boldsymbol{p})), \frac{(\boldsymbol{r} \cdot \boldsymbol{J})}{r^{5}}\right\}\right.\right. \\
\left.\left.+\left\{\boldsymbol{\Sigma} \cdot(\boldsymbol{p} \times(\boldsymbol{p} \times \boldsymbol{J})), \frac{1}{r^{3}}\right\}\right]\right\}-\frac{3 \hbar^{2} c^{2} G}{8}\left\{\left(5 p_{r}^{2}-\boldsymbol{p}^{2}\right) \frac{2 \epsilon^{2}+\epsilon m c^{2}+m^{2} c^{4}}{\epsilon^{4}\left(\epsilon+m c^{2}\right)^{2}}, \frac{(\boldsymbol{J} \cdot \boldsymbol{l})}{r^{5}}\right\},
\end{gathered}
$$

where $\boldsymbol{l}=\boldsymbol{r} \times \boldsymbol{p}$ is an angular momentum operator, and the operator $p_{r}^{2}=-\frac{\hbar^{2}}{r^{2}} \frac{\partial}{\partial r}\left(r^{2} \frac{\partial}{\partial r}\right)$ is proportional to the radial part of the Laplace operator. The equation of rotation of the spin is obtained via commuting the FW Hamiltonian with the polarization operator $\Pi=\beta \boldsymbol{\Sigma}$ and is given by

$$
\frac{d \boldsymbol{\Pi}}{d t}=\frac{i}{\hbar}\left[\mathcal{H}_{F W}, \boldsymbol{\Pi}\right]=\mathbf{\Omega}^{(1)} \times \boldsymbol{\Sigma}+\mathbf{\Omega}^{(2)} \times \boldsymbol{\Pi},
$$

where $\boldsymbol{\Omega}^{(1)}$ is the operator of angular velocity of rotation of the spin in the static gravitational field derived in Ref. [15],

$$
\boldsymbol{\Omega}^{(1)}=-\frac{m c^{4}}{\epsilon\left(\epsilon+m c^{2}\right)}(\boldsymbol{\phi} \times \boldsymbol{p})+\frac{c^{2}}{\epsilon}(\boldsymbol{f} \times \boldsymbol{p}),
$$

and the newly obtained contribution from the LT effect is equal to

$$
\begin{aligned}
\boldsymbol{\Omega}^{(2)}= & \frac{G}{c^{2} r^{3}}\left[\frac{3(\boldsymbol{r} \cdot \boldsymbol{J}) \boldsymbol{r}}{r^{2}}-\boldsymbol{J}\right]-\frac{3 G}{4}\left\{\frac{1}{\epsilon\left(\epsilon+m c^{2}\right)},\left[\frac{2\{\boldsymbol{l},(\boldsymbol{J} \cdot \boldsymbol{l})\}}{r^{5}}\right.\right. \\
& \left.\left.+\frac{1}{2}\left\{(\boldsymbol{p} \times \boldsymbol{l}-\boldsymbol{l} \times \boldsymbol{p}), \frac{(\boldsymbol{r} \cdot \boldsymbol{J})}{r^{5}}\right\}+\left\{(\boldsymbol{p} \times(\boldsymbol{p} \times \boldsymbol{J})), \frac{1}{r^{3}}\right\}\right]\right\} .
\end{aligned}
$$

The second term on the right-hand side of Eq. (22) contains an additional $\beta$ factor as compared to the first term. This is a manifestation of the gravitoelectric and the gravitomagnetic origin of the static gravitational field and of the Kerr (Lense-Thirring) field, 
respectively. The equation of spin motion in the electromagnetic field has a similar form (see Eq. (36) in Ref. [15]). The difference between the two terms on the right-hand side of Eq. (22) is caused by the fact that $\boldsymbol{\Omega}^{(1)}$ should contain the velocity operator rather than the momentum one. Since the velocity operator is proportional to an additional $\beta$ factor and is equal to $\boldsymbol{v}=\beta c \boldsymbol{p} / \epsilon$ for free particles, the operator $\boldsymbol{\Omega}^{(1)}$, expressed in terms of $\boldsymbol{v}$, also acquires an additional $\beta$ factor.

In Eq. (21), the Hamiltonian $\mathcal{H}_{F W}$ defines the zero component of the covariant fourmomentum operator, while its spatial components are expressed by the operator $\boldsymbol{p}$ taken with the opposite sign:

$$
p_{i}=i \hbar \frac{\partial}{\partial x^{i}}=\left(\frac{\mathcal{H}_{F W}}{c},-\boldsymbol{p}\right) .
$$

The equation of motion of the particle defines the evolution of the contravariant fourmomentum operator which spatial components $(a, b=1,2,3)$ are given by

$$
p^{a}=g^{a b} p_{b}+g^{0 a} p_{0}
$$

In a stationary metric, the evolution of the contravariant momentum operator in the weak field approximation is defined by

$$
F^{a}=\frac{d p^{a}}{d t}=-\frac{d p_{a}}{d t}+\frac{1}{4}\left\{\left\{v^{b}, \frac{\partial g^{a i}}{\partial x^{b}}\right\}, p_{i}\right\}, \quad \frac{d \boldsymbol{p}}{d t}=\frac{i}{\hbar}\left[\mathcal{H}_{F W}, \boldsymbol{p}\right],
$$

where $F^{a}$ is the force operator and $v^{a} \approx \beta c^{2} p^{a} / \epsilon \approx c^{2} p^{a} / \mathcal{H}_{F W}$ is the velocity operator.

One can calculate the force operator caused by the LT effect without allowance for contributions from $V, W$. This operator is equal to

$$
\boldsymbol{F}=\frac{c}{2}(\operatorname{curl} \boldsymbol{K} \times \boldsymbol{p}-\boldsymbol{p} \times \operatorname{curl} \boldsymbol{K})+\boldsymbol{F}_{s},
$$

where

$$
\begin{array}{r}
\operatorname{curl} \boldsymbol{K}=\frac{2 G}{c^{3} r^{3}}\left[\frac{3(\boldsymbol{r} \cdot \boldsymbol{J}) \boldsymbol{r}}{r^{2}}-\boldsymbol{J}\right], \quad \boldsymbol{F}_{s}=-\nabla\left(\frac{\hbar G}{2 c^{2} r^{3}}\left[\frac{3(\boldsymbol{r} \cdot \boldsymbol{J})(\boldsymbol{r} \cdot \boldsymbol{\Sigma})}{r^{2}}-\boldsymbol{J} \cdot \boldsymbol{\Sigma}\right]\right. \\
-\frac{3 \hbar G}{8}\left\{\frac{1}{\epsilon\left(\epsilon+m c^{2}\right)},\left[\frac{2\{(\boldsymbol{J} \cdot \boldsymbol{l}),(\boldsymbol{\Sigma} \cdot \boldsymbol{l})\}}{r^{5}}+\frac{1}{2}\left\{(\boldsymbol{\Sigma} \cdot(\boldsymbol{p} \times \boldsymbol{l})-\boldsymbol{\Sigma} \cdot(\boldsymbol{l} \times \boldsymbol{p})), \frac{(\boldsymbol{r} \cdot \boldsymbol{J})}{r^{5}}\right\}\right.\right. \\
\left.\left.\left.+\left\{\boldsymbol{\Sigma} \cdot(\boldsymbol{p} \times(\boldsymbol{p} \times \boldsymbol{J})), \frac{1}{r^{3}}\right\}\right]\right\}\right) .
\end{array}
$$

The operator equation (26) for the small spin-dependent force $\boldsymbol{F}_{s}$ is in the best compliance with the corresponding classical equation [10]. Since the Dirac spin operator is $s=\hbar \Sigma / 2$, the Eqs. (26), (27) yield the corresponding semiclassical equation:

$$
\mathcal{F}=c \operatorname{curl} \boldsymbol{K} \times \boldsymbol{p}+\mathcal{F}_{s}
$$




$$
\begin{array}{r}
\mathcal{F}_{s}=-\nabla\left(\frac{G}{c^{2} r^{3}}\left[\frac{3(\boldsymbol{r} \cdot \boldsymbol{J})(\boldsymbol{r} \cdot \boldsymbol{s})}{r^{2}}-\boldsymbol{J} \cdot \boldsymbol{s}\right]\right. \\
\left.-\frac{3 G}{\epsilon\left(\epsilon+m c^{2}\right)}\left[\frac{2(\boldsymbol{J} \cdot \boldsymbol{l})(\boldsymbol{s} \cdot \boldsymbol{l})}{r^{5}}+\frac{(\boldsymbol{s} \cdot[\boldsymbol{p} \times \boldsymbol{l}])(\boldsymbol{r} \cdot \boldsymbol{J})}{r^{5}}+\frac{(\boldsymbol{s} \cdot[\boldsymbol{p} \times[\boldsymbol{p} \times \boldsymbol{J}]])}{r^{3}}\right]\right) .
\end{array}
$$

Our relativistic result (27), (29) for the spin-dependent force perfectly agrees with the corresponding nonrelativistic classical formulas previously obtained in Ref. [16] on the basis of the Mathisson-Papapetrou equations [4, 5].

Our quantum Eqs. (26) -(29) actually agree with the classical results of both MathissonPapapetrou and Pomeransky-Khriplovich approaches. This follows from the fact that the spin-dependent part of Hamiltonian has the form $\mathcal{H}_{s}=\hbar\left(\boldsymbol{\Omega}^{(1)} \cdot \boldsymbol{\Sigma}+\boldsymbol{\Omega}^{(2)} \cdot \boldsymbol{\Pi}\right) / 2$ that perfectly agrees with the general classical Eq. (47) of the Ref. [7]. This is also in accordance with the earlier attempts (see Ref. [17], for example) to establish a direct general correspondence between the quantum dynamics and the classical equations of motion of the MathissonPapapetrou type.

The semiclassical formula corresponding to Eq. (24) and describing the motion of average spin has the form

$$
\boldsymbol{\Omega}^{(2)}=\frac{G}{c^{2} r^{3}}\left[\frac{3(\boldsymbol{r} \cdot \boldsymbol{J}) \boldsymbol{r}}{r^{2}}-\boldsymbol{J}\right]-\frac{3 G}{r^{3} \epsilon\left(\epsilon+m c^{2}\right)}\left[\frac{2 \boldsymbol{l}(\boldsymbol{J} \cdot \boldsymbol{l})+(\boldsymbol{p} \times \boldsymbol{l})(\boldsymbol{r} \cdot \boldsymbol{J})}{r^{2}}+\boldsymbol{p} \times(\boldsymbol{p} \times \boldsymbol{J})\right] .
$$

In a nonrelativistic approximation, the Eq. (30) coincides with the equation obtained by Schiff [2]. The second term in the Eq. (30) describes relativistic corrections. The Eq. (30) can also be expressed in the equivalent form:

$$
\boldsymbol{\Omega}^{(2)}=\frac{G}{c^{2} r^{3}}\left[\frac{3(\boldsymbol{r} \cdot \boldsymbol{J}) \boldsymbol{r}}{r^{2}}-\boldsymbol{J}\right]-\frac{3 G}{r^{5} \epsilon\left(\epsilon+m c^{2}\right)}[\boldsymbol{l}(\boldsymbol{l} \cdot \boldsymbol{J})+(\boldsymbol{r} \cdot \boldsymbol{p})(\boldsymbol{p} \times(\boldsymbol{r} \times \boldsymbol{J}))] .
$$

The quantum mechanical and semiclassical equations (21), (24), (26)-(31) are principal new results.

\section{CLASSICAL SPIN IN EXTERNAL FIELDS}

The dynamical equations that determine the motion of a spinning particle in external classical fields can be written, quite generally, in the form

$$
\begin{aligned}
\frac{d U^{\alpha}}{d \tau} & =\mathcal{F}^{\alpha}, \\
\frac{d S^{\alpha}}{d \tau} & =\Phi^{\alpha}{ }_{\beta} S^{\beta} .
\end{aligned}
$$


The forces $\mathcal{F}^{\alpha}$ are determined by the external fields (electromagnetic, gravitational, etc.) acting on a particle. Similarly, the spin is affected by the external fields through a spin transport matrix $\Phi^{\alpha}{ }_{\beta}$. Normalization of the velocity, $U_{\alpha} U^{\alpha}=c^{2}$, and its orthogonality to the spin, $S_{\alpha} U^{\alpha}=0$, impose on the right-hand sides of (32), (33) the conditions

$$
U_{\alpha} \mathcal{F}^{\alpha}=0, \quad U_{\alpha} \Phi^{\alpha}{ }_{\beta} S^{\beta}=S_{\alpha} \mathcal{F}^{\alpha}
$$

Since

$$
S_{\alpha} \Phi_{\beta}^{\alpha} S^{\beta}=\frac{1}{2} \frac{d\left(S_{\alpha} S^{\alpha}\right)}{d \tau}=0,
$$

the spin transport matrix is skew-symmetric: $\Phi_{\alpha \beta}=-\Phi_{\beta \alpha}$.

The components of the 4-velocity are conveniently parametrized by the spatial 3-velocity $v^{a}(a=1,2,3)$ as

$$
U^{\alpha}=\left(\begin{array}{c}
\gamma \\
\gamma v^{a}
\end{array}\right)
$$

where $\gamma=\left(1-v^{2} / c^{2}\right)^{-1 / 2}$ is the Lorentz factor $\left(v^{2}=\delta_{a b} v^{a} v^{b}\right)$. When the particle is at rest, $v^{a}=0$, its 4 -velocity reduces to

$$
u^{\alpha}=\delta_{0}^{\alpha}=\left(\begin{array}{l}
1 \\
0
\end{array}\right) .
$$

The actual 4-velocity $U^{\alpha}$ is obtained from the rest-frame components with the help of the Lorentz transformation $U^{\alpha}=\Lambda_{\beta}^{\alpha} u^{\beta}$ where

$$
\Lambda_{\beta}^{\alpha}=\left(\begin{array}{c|c}
\gamma & \gamma v_{b} / c^{2} \\
\hline \gamma v^{a} & \delta_{b}^{a}+(\gamma-1) v^{a} v_{b} / v^{2}
\end{array}\right) .
$$

Hereafter the Latin indices from the beginning of the alphabet $(a, b, \cdots=1,2,3$ which label the spatial components of the objects) are raised and lowered with the help of the Euclidean 3-dimensional metric $\delta_{a b}$.

The physical components of spin $s^{\alpha}$ are defined in the rest frame of a particle. Accordingly, we have $S^{\alpha}=\Lambda_{\beta}^{\alpha} s^{\beta}$. The dynamical equation for the physical spin is derived by substituting this relation into (33) which yields

$$
\frac{d s^{\alpha}}{d \tau}=\Omega_{\beta}^{\alpha} s^{\beta}
$$

Here we introduced $\Omega^{\alpha}{ }_{\beta}=\phi_{\beta}^{\alpha}+\pi_{\beta}^{\alpha}$ where

$$
\phi_{\beta}^{\alpha}=\left(\Lambda^{-1}\right)^{\alpha}{ }_{\gamma} \Phi_{\delta}^{\gamma} \Lambda_{\beta}^{\delta}, \quad \pi_{\beta}^{\alpha}=-\left(\Lambda^{-1}\right)^{\alpha}{ }_{\gamma} \frac{d}{d \tau} \Lambda_{\beta}^{\gamma} .
$$


The physical spin has only three spatial components. One can verify that the 0 -th component of (39) is identically satisfied [in fact, it is identical to the second compatibility condition (34)]. As a result, the dynamical equation (39) reduces to the 3 -vector form

$$
\frac{d s^{a}}{d \tau}=\Omega^{a}{ }_{b} s^{b}, \quad \text { or } \quad \frac{d s}{d \tau}=\Omega \times s .
$$

Here $\boldsymbol{\Omega}=\boldsymbol{\phi}+\boldsymbol{\pi}$ and the components of the 3 -vectors are introduced by $\boldsymbol{s}=\left(s^{1}, s^{2}, s^{3}\right)$ and $\Omega=\left(\Omega_{32}, \Omega_{13}, \Omega_{21}\right)$.

The new general equations (40)-(41) are valid for a spinning particle interacting with any external fields. In the next section, we specify these equations to the case of the gravitational field.

\section{EQUIVALENCE PRINCIPLE AND DYNAMICS OF CLASSICAL AND QUANTUM SPINS IN CURVED SPACETIMES}

The equivalence principle (EP) is known to be the cornerstone of general relativity. The EP results in the general equation of motion of classical test particles in curved spacetimes:

$$
\frac{D U^{\alpha}}{d \tau}=0
$$

where $D /(d \tau)$ denotes the covariant derivative along the curve. The corresponding equation of motion of the four-component spin used in Refs. [7, 8] and many other works is very similar:

$$
\frac{D S^{\alpha}}{d \tau}=0
$$

In the present work, we do not consider a relatively weak influence of the spin on particle's trajectory produced by the Mathisson-Papapetrou force [4, 5, 7] which results in a weak violation of the equivalence principle by the curvature-dependent terms [18]. For the Kerr spacetime, the deviation from the geodetic motion under the influence of spin was recently comprehensively studied in the framework of the analytic perturbation approach in [19], see also the relevant references there.

In the context of our present investigation of the dynamics of spin, it is worthwhile to stress that the account of the Mathisson-Papapetrou terms does not change the spin dynamics in the current approximation. There is thus no any difference between the MathissonPapapetrou and Pomeransky-Khriplovich approaches within our framework. Nevertheless 
we find it more convenient to refer specifically to [7] where the exact equation of motion for the three-component spin was obtained in explicit form.

The Eq. (42) states identical motion of all classical particles in curved spacetimes. Similarly, the Eq. (43) states identical motion of all classical spins (gyroscopes). This important conclusion leads to a great difference between dynamics of the spin in electrodynamics and gravity. Angular velocities of precession of all classical and quantum spins moving with the same velocity in the curved spacetime are equal. Thus, spinning particles cannot have any anomalous gravitomagnetic moments [20]. It has been proved that both the anomalous gravitomagnetic moment and the gravitoelectric dipole moment being gravitational analogs of the anomalous magnetic moment and the electric dipole moment, respectively, are identically zero [20]. Relations obtained by Kobzarev and Okun predict equal frequencies of precession of classical and quantum spins in any curved spacetimes [21, 22]. Nevertheless, this conclusion was discussed for a long time (see Refs. [15, 22, 23, 24] and references therein).

On the contrary, angular velocities of spin precession of different particles which are determined by the Thomas-Bargmann-Michel-Telegdi equation [25, 26] do not coincide and, generally speaking, differ from an angular velocity of precession of a classical rotator.

Comparing (42)-(43) with (32) and (33), we find the explicit expressions for the force and the spin transport matrix

$$
\mathcal{F}^{\alpha}=\Phi^{\alpha}{ }_{\beta} U^{\beta}, \quad \Phi_{\beta}^{\alpha}=-U^{i} \Gamma_{i \beta}{ }^{\alpha},
$$

in terms of the gravitational field $\Gamma_{i \beta}{ }^{\alpha}$. Using this in (40), we find explicitly

$$
\begin{aligned}
\phi^{a b} & =-U^{i}\left[\Gamma_{i}^{b a}+\frac{\gamma^{2}}{\gamma+1} \frac{v_{c}}{c^{2}}\left(\Gamma_{i c}{ }^{a} v^{b}-\Gamma_{i c}{ }^{b} v^{a}\right)+\frac{\gamma}{c^{2}}\left(\Gamma_{i \widehat{0}}{ }^{a} v^{b}-\Gamma_{i \widehat{0}}{ }^{b} v^{a}\right)\right], \\
\pi^{a b} & =U^{i} \frac{\gamma^{2}}{\gamma+1}\left[\frac{v_{c}}{c^{2}}\left(\Gamma_{i c}{ }^{a} v^{b}-\Gamma_{i c}{ }^{b} v^{a}\right)+\frac{1}{c^{2}}\left(\Gamma_{i \widehat{0}}{ }^{a} v^{b}-\Gamma_{i \widehat{0}} b v^{a}\right)\right] .
\end{aligned}
$$

Hence the precession of the physical spin in the gravitational field is described by

$$
\Omega_{a}=\epsilon_{a b c} U^{i}\left(\frac{1}{2} \Gamma_{i}{ }^{c b}+\frac{\gamma}{\gamma+1} \Gamma_{i 0}{ }^{b} v^{c} / c^{2}\right) .
$$

This exact formula can be used also in the flat spacetime for noninertial reference frames, since the connection $\Gamma_{i \beta}{ }^{\alpha}$ contains information about both gravitational and inertial effects.

The Eq. (47) has been first obtained by Pomeransky and Khriplovich [7] as a result of a comparison of the equations of motion of spin in electrodynamics and gravity, and more 
recently has been consistently derived by Dvornikov [8]. Note that unlike Ref. [8], our results can be easily extended to any external fields (electromagnetic, gravitational, scalar, and other).

\section{CLASSICAL SPIN IN NONSTATIC SPACETIMES}

Description of a spin requires the introduction of a tetrad (the frame $e_{\alpha}=e_{\alpha}^{i} \partial_{i}$ and the dual coframe $\vartheta^{\alpha}=e_{i}^{\alpha} d x^{i}$ ). In physical terms a choice of a tetrad means a selection of a local reference system of an observer.

Mathematically, there are infinitely many tetrads since a reference frame of an observer can obviously be constructed in an infinitely many ways. In particular, from a given tetrad field $e_{i}^{\alpha}$ we can obtain a continuous family of tetrads by performing the Lorentz transformation $e_{i}^{\prime \alpha}=\Lambda_{\beta}^{\alpha} e_{i}^{\beta}$, where the elements of the Lorentz matrix $\Lambda_{\beta}^{\alpha}(x)$ are arbitrary functions of the spacetime coordinates. In practice, there are three most widely used gauges.

Schwinger gauge. Probably for the first time introduced independently by Schwinger [27] and Dirac [28] (and widely used in many works, including [9] and our current study), this choice demands that the tetrad matrix $e_{i}^{\alpha}$, and its inverse $e_{\alpha}^{i}$, both have the trivial elements in the upper-right blocks:

$$
e_{i}^{\alpha}=\left(\begin{array}{c|c}
e_{0}^{\widehat{0}} & 0 \\
\hline e_{0}^{\widehat{a}} & e_{b}^{\widehat{a}}
\end{array}\right), \quad e_{\alpha}^{i}=\left(\begin{array}{c|c}
e_{\widehat{0}}^{0} & 0 \\
\hline e_{\widehat{0}}^{a} & e_{\widehat{b}}^{a}
\end{array}\right)
$$

Landau-Lifshitz gauge (see, e.g., Ref. [29]) fixes the tetrad matrices so that they both have the trivial elements in the lower-left blocks:

$$
e_{i}^{\alpha}=\left(\begin{array}{c|c}
e_{0}^{\widehat{0}} & e_{b}^{\widehat{0}} \\
\hline 0 & e_{b}^{\widehat{a}}
\end{array}\right), \quad e_{\alpha}^{i}=\left(\begin{array}{c|c}
e_{\widehat{0}}^{0} & e_{\widehat{b}}^{0} \\
\hline 0 & e_{\widehat{b}}^{a}
\end{array}\right) .
$$

Symmetric gauge. Using the Minkowski flat metric $g_{\alpha \beta}=\operatorname{diag}\left(c^{2},-1,-1,-1\right)$, we can move the anholonomic index down and construct the matrix $e_{\alpha i}:=g_{\alpha \beta} e_{i}^{\beta}$. The tetrad is called symmetric (hence the name, symmetric gauge) when the resulting matrix is invariant under the transposition operation which we symbolically can write as

$$
e_{\alpha i}=e_{i \alpha}
$$

Such a tetrad was used by Pomeransky and Khriplovich [7] and Dvornikov [8]. 
In the framework of our current study, we choose the Schwinger gauge by specifying the coframe as (10).

The other tetrads are obtained from our $e_{i}^{\alpha}$ with the help of the Lorentz transformation $e_{i}^{\prime \alpha}=\Lambda^{\alpha}{ }_{\beta} e_{i}^{\beta}$, where

$$
\Lambda_{\beta}^{\alpha}=\left(\begin{array}{c|c}
\lambda & \lambda q_{b} / c \\
\hline \lambda c q^{a} & \delta_{b}^{a}+(\lambda-1) q^{a} q_{b} / q^{2}
\end{array}\right) .
$$

Here we denote

$$
q^{a}=\xi \frac{W K^{a}}{V}, \quad \lambda=\frac{1}{\sqrt{1-q^{2}}} .
$$

(As usual, $q^{2}=\delta_{a b} q^{a} q^{b}$ ). The constant $\xi$ conveniently parametrizes different choices of tetrads. Namely, for $\xi=1 / 2$ the Lorentz matrix (51) transforms our tetrad to that of Pomeransky and Khriplovich, and for $\xi=1$ we obtain the tetrad of Landau and Lifshitz.

Under the Lorentz transformation $e_{i}^{\prime \alpha}=\Lambda^{\alpha}{ }_{\beta} e_{i}^{\beta}$, the connection changes from $\Gamma_{i \alpha}{ }^{\beta}$ to the new one: $\Gamma_{i \alpha}^{\prime}{ }^{\beta}=\Lambda^{\alpha}{ }_{\gamma} \Gamma_{i \delta}{ }^{\gamma}\left(\Lambda^{-1}\right)^{\delta}{ }_{\beta}+\Lambda_{\gamma}^{\alpha} \partial_{i}\left(\Lambda^{-1}\right)^{\gamma}{ }_{\beta}$. Specifically, for the weak gravitational field of a slowly rotating source (3) we find

$$
\begin{aligned}
& \Gamma_{i \widehat{a}}^{\prime \widehat{0}}=\frac{G M x_{a}}{c^{2} r^{3}} e_{i}^{\prime \widehat{0}}-\frac{3 x_{(a} K_{b)}}{c r^{2}} e_{i}^{\prime \widehat{b}}+\xi \frac{\epsilon_{a b c} \omega^{b}}{c^{2}}\left(-\delta_{d}^{c}+\frac{3 x^{c} x_{d}}{r^{2}}\right) e_{i}^{\prime \widehat{d}}, \\
& \Gamma_{\hat{i} \widehat{a}}^{\prime \widehat{a}}=\frac{1}{2} \epsilon_{a b c} \omega^{d}\left(-\delta_{d}^{c}+\frac{3 x^{c} x_{d}}{r^{2}}\right) e_{i}^{\prime \widehat{0}}+\frac{G M}{c^{2} r^{3}}\left(x^{a} e_{i}^{\prime \widehat{b}}-x^{b} e_{i}^{\prime \widehat{a}}\right) .
\end{aligned}
$$

Recall that $\Gamma_{i \widehat{0}}^{\prime \widehat{a}}=c^{2} \delta^{a b} \Gamma_{i \widehat{b}}^{\prime \widehat{ }}$. We can drop the primes now, since the value of the $\xi$ parameter identifies the reference frame anyway.

Substituting (53) and (54) into (47), we obtain the precession of the physical spin in the gravitational field of rotating object:

$$
\boldsymbol{\Omega}=\gamma\left\{\frac{G}{c^{2} r^{3}}\left[\frac{3 \boldsymbol{r}(\boldsymbol{r} \cdot \boldsymbol{J})}{r^{2}}-\boldsymbol{J}\right]+\frac{\boldsymbol{\rho} \times \boldsymbol{v}}{c^{2}}\right\},
$$

where we denote

$$
\boldsymbol{\rho}=\frac{2 \gamma+1}{\gamma+1} \frac{G M}{r^{3}} \boldsymbol{r}+\frac{\gamma}{\gamma+1} \frac{3 G}{c^{2} r^{3}}\left[\frac{\boldsymbol{r}}{r^{2}}(\boldsymbol{r} \cdot(\boldsymbol{J} \times \boldsymbol{v}))-\frac{2 \xi}{3} \boldsymbol{J} \times \boldsymbol{v}+(2 \xi-1) \frac{(\boldsymbol{r} \cdot \boldsymbol{v})}{r^{2}} \boldsymbol{J} \times \boldsymbol{r}\right] .
$$

Putting $\xi=0$, thus specifying to the Schwinger tetrad, we find that the classical formula (55) perfectly reproduces the quantum result (31). The extra Lorentz factor is due to the fact that the classical evolution of spin was measured by using the proper time $\tau$, whereas the quantum evolution was analyzed by using the coordinate time $t$. If we choose another 
tetrad by putting $\xi=1 / 2$ in (包), the equation (55) yields the result by Pomeransky and Khriplovich [7] and Dvornikov [8]:

$$
\boldsymbol{\Omega}^{(P K)}=\frac{G}{c^{2} r^{3}}\left[\frac{3(\boldsymbol{r} \cdot \boldsymbol{J}) \boldsymbol{r}}{r^{2}}-\boldsymbol{J}\right]-\frac{\gamma}{\gamma+1} \frac{G}{c^{2} r^{3}}\left\{\frac{3[\boldsymbol{r} \times \boldsymbol{v}](\boldsymbol{J} \cdot[\boldsymbol{r} \times \boldsymbol{v}])}{r^{2}}+\boldsymbol{v} \times(\boldsymbol{v} \times \boldsymbol{J})\right\} .
$$

This result evidently differs from the Eq. (31).

It is worthwhile to mention that our result (55)-(56) (together with its quantum (31) counterpart) presented in Ref. [38] was confirmed in the recent paper [39] (specifically, cf. the eq. (6.19) therein). This is very satisfactory, since the authors of [39] worked in a different framework developing the Hamiltonian theory of a spinning particle in a curved spacetime. In Ref. [39], the first relativistic corrections were calculated, while our Eqs. (55) -(56) and the corresponding quantum equations are the exact formulas suitable also for the discussion of an ultrarelativistic spin-1/2 particle. It is also stressed in [39] that the

results obtained are consistent, in the test-particle limit, with the earlier analysis [40] of the dynamics of two gravitationally interacting rotating extended bodies.

\section{PARTICLE WITH SPIN IN A ROTATING FRAME}

One can straightforwardly show that Eq. (47) yields the correct angular velocity of spin precession in a rotating frame. The spacetime is flat in this case with the line element [9] given by

$$
d s^{2}=\left[c^{2}-(\boldsymbol{\omega} \times \boldsymbol{r})^{2}\right] d t^{2}-2(\boldsymbol{\omega} \times \boldsymbol{r})_{a} d x^{a} d t-\left(d x^{a}\right)^{2} .
$$

We choose the Schwinger gauge for the tetrad, which is then described by (10) with (8) , where we have to put $\boldsymbol{a}=0$ for the pure rotation. Other tetrads are easily obtained with the help of the Lorentz transformation (51) which is much simpler now because $V=W=1$. One can verify that the choosing $\xi=1 / 2$ we indeed obtain a symmetric tetrad (which corresponds to the gauge of Pomeransky and Khriplovich), whereas $\xi=1$ yields the Landau-Lifshitz tetrad.

The corresponding family of the (transformed) connection reads

$$
\Gamma_{i \widehat{a}}^{\prime \widehat{0}}=-\frac{\xi}{c^{2}} \epsilon_{a b c} \omega^{c} e_{i}^{\prime \widehat{b}}, \quad \Gamma_{i \widehat{b}}^{\prime \widehat{a}}=-\epsilon_{b a c} \omega^{c} e_{i}^{\prime \widehat{0}}
$$


Substituting this into the eq. (47), we find the precession angular velocity

$$
\boldsymbol{\Omega}=\gamma\left(-\boldsymbol{\omega}+\frac{\xi \gamma}{\gamma+1} \frac{\boldsymbol{v} \times(\boldsymbol{v} \times \boldsymbol{\omega})}{c^{2}}\right)
$$

The overall Lorentz factor is again due to the use of the proper time in the evolution equations.

The correct result for the Schwinger gauge (that is recovered for $\xi=0$ ) was first obtained in [30, 31]. A transparent and simple explanation of the dependence of the angular velocity of the spin precession on the gauge of a tetrad, and thus of the additional terms which are present in (60) in the symmetric gauge (for $\xi=1 / 2$ ) and in the Landau-Lifshitz gauge (for $\xi=1$ ), was presented recently [23] on the basis of the Thomas precession.

The study of a rotating frame helps to clarify the difference between the tetrad gauges. The line element (58) describes the flat spacetime. Indeed, we can bring the metric to the explicitly flat form by a coordinate transformation that replaces $\left(t, x^{a}\right)$ with the new coordinates $\left(T, X^{a}\right)$ using the formulas

$$
t=T, \quad x^{a}=L_{b}^{a} X^{b} .
$$

Here the $3 \times 3$ matrix

$$
L_{b}^{a}=n^{a} n_{b}+\left(\delta_{b}^{a}-n^{a} n_{b}\right) \cos \varphi+\epsilon_{c b}^{a} n^{c} \sin \varphi
$$

defines a rotation around the unit vector $n^{a}$ on an angle $\varphi(T)=\omega T$ with the constant angular velocity $\dot{\varphi}=\omega$, where $\omega_{a}=\omega n_{a}$. Differentiating (61), we easily verify

$$
d x^{a}-K^{a} c d t=L_{b}^{a} d X^{a} .
$$

Accordingly, the transformation (61) brings the line element (58) to $d s^{2}=c^{2} d T^{2}-$ $\delta_{a b} d X^{a} d X^{b}$ which is the flat Minkowski world described in the Cartesian coordinates $\left(T, X^{a}\right)$.

In order to compare different tetrad gauges, let us consider the continuous family that arises from the Lorentz transformation (51). Explicitly, the tetrad components read

$$
e_{i}^{\alpha}=\left(\begin{array}{c|c}
e_{0}^{\widehat{0}}=\lambda\left(1-\xi K^{2}\right) & e_{b}^{\widehat{0}}=\lambda \xi K_{b} / c \\
\hline e_{0}^{\widehat{a}}=\lambda(\xi-1) c K^{a} & e_{b}^{\widehat{a}}=\delta_{b}^{a}+(\lambda-1) K^{a} K_{b} / K^{2}
\end{array}\right) .
$$

Here, as before, $\lambda=1 / \sqrt{1-\xi^{2} K^{2}}$. As we can easily verify, this family indeed contains all the three main options: (i) for $\xi=0$, the Schwinger gauge is obtained, (ii) the LandauLifshitz gauge arises for $\xi=1$, (iii) the symmetric gauge is recovered for $\xi=1 / 2$. 
Let us now analyse a particle that is at rest with respect to a reference system described by the tetrad (64). The tetrad components of the 4 -velocity of such a particle read $U^{\alpha}=$ $\delta_{\hat{0}}^{\alpha}=(1, \mathbf{0})$. Respectively, the world components of particle's 4 -velocity read $d x^{i} / d \tau=U^{i}=$ $e_{\alpha}^{i} U^{\alpha}=e_{\hat{0}}^{i}$. Explicitly we then find from the inverse of (64)

$$
\frac{d t}{d \tau}=\lambda, \quad \frac{d x^{a}}{d \tau}=\lambda c(1-\xi) K^{a} \quad \Longrightarrow \quad \frac{d x^{a}}{d t}=(1-\xi) \epsilon^{a b c} \omega_{b} x_{c} .
$$

This is how particle's dynamics is described in the coordinates $\left(t, x^{a}\right)$ of a homogeneously rotating flat world. But how does this motion look in terms of the genuinely inertial coordinates? Substituting (61) into (65), we obtain

$$
\frac{d X^{a}}{d T}=-\xi \epsilon^{a b c} \omega_{b} X_{c}
$$

As we see, for $\xi=0$ the particle is at rest in the inertial coordinates, $X^{a}=$ const. That is, a particle which is at rest in the Schwinger reference frame indeed does not physically move in the Cartesian coordinates $\left(T, X^{a}\right)$. However, when a particle is at rest with respect to the Landau-Lifshitz $\xi=1$ tetrad or with respect to the symmetric $\xi=1 / 2$ tetrad, it actually turns out to be moving (rotating) in the inertial coordinates!

In this sense, the Schwinger gauge is physically distinguished as it qualifies for defining an almost inertial reference frame. In all the other tetrads with $\xi \neq 0$, the description of physical effects is "spoiled" by non-inertiality. Such a spoiling effect is manifested by the additional (last) term in (60), for example.

Certainly, one should be careful when generalizing the above observation to the case of the nontrivial gravitational field. Note that we use the expression "almost inertial" to distinguish the anholonomic Schwinger tetrad from a truly inertial tetrad which is holonomic. In a curved spacetime one cannot, as a matter of principle, separate the inertial effects from the gravitational ones. However, we recall again that Lense and Thirring have demonstrated, in the weak-field approximation, the similarity of particle's motion in a gravitational field of a rotating source to its dynamics in a noninertial frame rotating with the angular velocity (99). Our analysis thus demonstrates that the Schwinger choice clearly appears to be preferable.

\section{DISCUSSION AND CONCLUSION}

In this paper, we consider the quantum and classical dynamics of a particle with spin in the gravitational field of a rotating source. Being primarily interested in the dynamics 
of spin, we derive the quantum-mechanical and semiclassical equations of motion of the spin of a Dirac particle from the Foldy-Wouthuysen approach. We demonstrate that the precession of the quantum spin is in a perfect agreement with the motion of the classical spin derived within a general scheme of Sec. IV The results obtained are compared to the earlier computations for different tetrad gauges, and we show that the precession effect depends crucially on the choice of a tetrad. At the same time, we find a perfect consistency with the classical Mathisson-Papapetrou approach by explicitly calculating the quantum and semiclassical expressions for the spin-dependent force on a Dirac particle.

The Lense-Thirring effect or frame dragging is one of the most impressive predictions of the general relativity. This effect is currently analyzed in the Gravity Probe B experiment [32, 33]. However, relativistic corrections to the LT effect are not observable in this experiment as well as in other experiments inside the solar system [34].

Nevertheless, it is necessary to take the relativistic corrections to the LT precession into account for the investigation of physical phenomena in the binary stars such as pulsar systems. In this case, both components of a system undergo a mutual Lense-Thirring precession about the total angular momentum $\boldsymbol{J}$. Since the spin precession effects are well observable [35, 36, 37], the use of the results obtained in the present work may be helpful for the high-precision calculations of spin dynamics in the binaries.

\section{Acknowledgments}

We are grateful to Lewis Ryder and Gerhard Schäfer for the useful discussions and correspodence. This work was supported in part by the Belarusian Republican Foundation for Fundamental Research (grant No. 908D-001), the program of collaboration BLTP/Belarus, the Deutsche Forschungsgemeinschaft (grants No. HE 528/21-1 and No. 436 RUS 113/881/0), the Russian Foundation for Basic Research (grants No. 09-02-01149 and No. 09-01-12179), and the Russian Federation Ministry of Education and Science (grant No. MIREA 2.2.2.2.6546).

[1] H. Thirring, Über die Wirkung rotierender ferner Massen in der Einsteinschen Gravitationstheorie, Phys. Z. 19 (1918) 33-39 [English translation: On the effect of rotating distant masses 
in Einstein's theory of gravitation], Gen. Rel. Grav. 16 (1984) 712-725]; H. Thirring, Berichtigung zu meiner Arbeit: "Über die Wirkung rotierender ferner Massen in der Einsteinschen Gravitationstheorie", Phys. Z. 22 (1921) 29-30 [English translation: Correction to my paper: "On the effect of rotating distant masses in Einstein's theory of gravitation", Gen. Rel. Grav. 16 (1984) 725-727]; J. Lense and H. Thirring, Über den Einfluß der Eigenrotation der Zentralkörper auf die Bewegung der Planeten und Monde nach der Einsteinschen Gravitationstheorie, Phys. Z. 19 (1918) 156-163 [English translation: On the influence of the proper rotation of central bodies on the motions of planets and moons according to Einstein's theory of gravitation, Gen. Rel. Grav. 16 (1984) 727-750].

[2] L.I. Schiff, On experimental tests of the general theory of relativity, Am. J. Phys. 28 (1960) 340343; L.I. Schiff, Motion of gyroscope according to Einstein's theory of gravitation, Proc. Nat. Acad. Sci. 46 (1960) 871-882; L.I. Schiff, Possible new experimental test of general relativity theory, Phys. Rev. Lett. 4 (1960) 215-217.

[3] I.Yu. Kobzarev and V.I. Zakharov, Spin precession in a gravitational field, Ann. Phys. (USA) 37 (1966) 1-6; R.F. O'Connell, Schiff's proposed gyroscope experiment as a test of the scalartensor theory of general relativity, Phys. Rev. Lett. 20 (1968) 69-71; D.C. Wilkins, General equation for the precession of a gyroscope, Ann. Phys. (USA) 61 (1970) 277-293; B. Mashhoon, Partics with spin in a gravitational field, J. Math. Phys. 12 (1971) 1075-1077.

[4] M. Mathisson, Neue Mechanik materieller Systeme, Acta Phys. Polon. 6 (1937) 163-200.

[5] A. Papapetrou, Spinning test particles in general relativity. I, Proc. Roy. Soc. Lond. A 209 (1951) 248-258.

[6] H.C. Corben, Classical and quantum theories of spinning particles (Holden-Day, Inc: San Francisco, 1968) 279 pp.; B.M. Barker and R.F. O'Connell, The gravitational interaction: Spin, rotation, and quantum effects - A review, Gen. Rel. Grav. 11 (1979) 149-175.

[7] A.A. Pomeransky and I.B. Khriplovich, Equations of motion of spinning relativistic particle in external fields, Zh. Eksp. Teor. Fiz. 113 (1998) 1537-1557 [J. Exp. Theor. Phys. 86 (1998) 839-849].

[8] M. Dvornikov, Neutrino spin oscillations in gravitational field, Int. J. Mod. Phys. D 15 (2006) 1017-1033; hep-ph/0601095

[9] F.W. Hehl and W.T. Ni, Inertial effects of a Dirac particle, Phys. Rev. D 42 (1990) 2045-2048.

[10] L.D. Landau and E.M. Lifshitz, The Classical Theory of Fields, 4th revised English edition 
(Butterworth-Heinemann, Oxford, 1980) Sec. 88, p. 272.

[11] Yu.N. Obukhov, Spin, gravity, and inertia, Phys. Rev. Lett. 86 (2001) 192-195.

[12] Yu.N. Obukhov, On gravitational interactions of fermions, Fortschr. Phys. 50 (2002) 711-716.

[13] A.J. Silenko, Foldy-Wouthuysen transformation forrelativistic particles in external fields, J. Math. Phys. 44 (2003) 2952-2966.

[14] A.J. Silenko, Foldy-Wouthyusen transformation and semiclassical limit for relativistic particles in strong external fields, Phys. Rev. A 77 (2008) 012116 [7 pages].

[15] A.J. Silenko and O.V. Teryaev, Semiclassical limit for Dirac particles interacting with a gravitational field, Phys. Rev. D 71 (2005) 064016 [8 pages].

[16] R. Wald, Gravitational spin interaction, Phys. Rev. D 6 (1972) 406-413; B.M. Barker and R.F. O'Connell, The gravitational interactions: Spin, rotation, and quantum effects - A review, Gen. Rel. Grav. 11 (1979) 149-175.

[17] S.K. Wong, Heisenberg equations of motion for spin-1/2 wave equation in general relativity, Int. J. Theor. Phys. 5 (1972) 221-230; L. Kannenberg, Mean motion of Dirac electrons in a gravitational field, Ann. Phys (USA) 103 (1977) 64-73; J. Audretsch and D.J. Diestler, Trajectories and spin motion of massive spin-1/2 particles in gravitational fields, J. Phys. A: Math. and Gen. A14 (1981) 411-422.

[18] R. Plyatsko, Gravitational ultrarelativistic spin-orbit interaction and the weak equivalence principle, Phys. Rev. D 58 (1998) 084031 [5 pages]; R. Plyatsko, Fermi-transported spinor and Dirac equation in general relativity, arXiv: gr-qc/0601111 (2006).

[19] B. Mashhoon and D. Singh, Dynamics of extended spinning masses in a gravitational field, Phys. Rev. D 74 (2006) [12 pages]; D. Singh, Perturbation method for classical spinning particle motion. I. Kerr space-time, Phys. Rev. D 78 (2008) [21 pages]; D. Singh, An analytic perturbation approach for classical spinning particle dynamics, Gen. Rel. Grav. 40 (2008) 1179-1192.

[20] I.Yu. Kobzarev and L.B. Okun, Gravitational interaction of fermions, Zh. Eksp. Teor. Fiz. 43 (1962) 1904-1909 [Sov. Phys. JETP 16 (1963) 1343-1346].

[21] O.V. Teryaev, Spin structure of nucleon and equivalence principle, arXiv: hep-ph/9904376 (1999); O.V. Teryaev, Sources of time reversal odd spin asymmetries in QCD, arXiv: hep-ph/0306301 (2003).

[22] A.J. Silenko and O.V. Teryaev, Equivalence principle and experimental tests of gravitational 
spin effects, Phys. Rev. D 76 (2007) 061101(R) [5 pages].

[23] A.J. Silenko, Classical and quantum spins in curved spacetimes, Acta Phys. Polon. B Proc. Suppl. 1 (2008) 87-107.

[24] B. Mashhoon, Quantum theory in accelerated frames of reference, Lect. Notes Phys. 702 (2006) 112-132.

[25] V. Bargmann, L. Michel, and V.L. Telegdi, Precession of the polarization of particles moving in a homogeneous electromagnetic field, Phys. Rev. Lett. 2 (1959) 435-436.

[26] L.H. Thomas, The motion of the spinning electron, Nature (London) 117 (1926) 514; L.H. Thomas, The kinematics of an electron with an axis, Phil. Mag. (ser. 7) 3 (1927) 122 .

[27] J. Schwinger, Energy and momentum density in field theory, Phys. Rev. 130 (1963) 800-805; J. Schwinger, Quantized gravitational field, Phys. Rev. 130 (1963) 1253-1258.

[28] P.A.M. Dirac, Interacting gravitational and spinor fields, in: "Recent developments in general relativity" (Pergamon Press, Oxford and PWN, Warsaw, 1962) pp. 191-200.

[29] Ref. [10], Sec. 98.

[30] A. Gorbatsevich, Exp. Tech. Phys. 27, 529 (1979).

[31] B. Mashhoon, Neutron interferometry in a rotating frame of reference, Phys. Rev. Lett. 61 (1988) 2639-2642.

[32] I. Ciufolini and E.C. Pavlis, A confirmation of the general relativistic prediction of the LenseThirring effect, Nature 431 (2004) 958-960.

[33] C.W.F. Everitt et al., Gravity Probe B data analysis status and potential for improved accuracy of scientific results, Class. Quantum Grav. 25 (2008) 114002 (11 pages).

[34] L. Iorio, Testing frame-dragging with the Mars Global Surveyor spacecraft in the gravitational field of Mars, in The measurement of gravitomagnetism: A challenging enterprise, ed. by L. Iorio (Nova publishers, Hauppauge (NY), 2007), pp. 177-187; L. Iorio and V. Lainey, The Lense-Thirring effect in the Jovian system of the Galilean satellites and its measurability, Int. J. Mod. Phys. D 14 (2005) 2039-2050.

[35] R.D. Blandford, Lense-Thirring precession of radio pulsars, J. Astrophys. Astron., 16 (1995) 191-206.

[36] L. Stella and M. Vietri, Lense-Thirring precession and quasi-periodic oscillations inlow-mass X-ray binaries, Astrophys. J. 492 (1998) L59-L62. 
[37] A. W. Hotan, M. Bailes, and S. M. Ord, Geodetic precession in PSR J1141-6545, Astrophys. J. 624 (2005) 906-913.

[38] Yu.N. Obukhov, A.J. Silenko, and O.V. Teryaev, Classical and quantum equations of motion of spin for particles in nonstatic spacetimes, in: Proc. of XIII Internat. Conf. "Selected Problems of Modern Physics", Dubna, 23-27 June 2008, Eds. B.M. Barbashov and S.M. Eliseev (Joint Inst. Nucl. Res., JINR, Dubna, 2009) pp. 168-170; Yu.N. Obukhov, A.J. Silenko, and O.V. Teryaev, Spin dynamics in gravitational fields of rotating bodies and the equivalence principle, arXiv: 0907.4367 (gr-qc).

[39] E. Barausse, E. Racine, and A. Buonanno, Hamiltonian of a spinning test-particle in curved spacetime, arXiv: 0907.4745 (gr-qc).

[40] J. Steinhoff, S. Hergt, and G. Schäfer, ADM canonical formalism for gravitating spinning objects, Phys. Rev. D 77 (2008) 104018 [16 pages]; J. Steinhoff, S. Hergt, and G. Schäfer, Spin-squared Hamiltonian of next-to-leading order gravitational interaction, Phys. Rev. D $\mathbf{7 8}$ (2008) 101503 [5 pages]. 